\title{
VL: A further case of erroneous recollection
}

\author{
Fergus I.M. Craik ${ }^{\mathrm{a}, \mathrm{b}, *}$, Morgan D. Barense ${ }^{\mathrm{a}, \mathrm{b}}$, Clare J. Rathbone ${ }^{\mathrm{a}, \mathrm{c}}$, Joan E. Grusec ${ }^{\mathrm{b}}$, \\ Donald T. Stuss $^{\mathrm{a}, \mathrm{b}}$, Fuqiang Gao ${ }^{\mathrm{d}}$, Christopher J.M. Scott ${ }^{\mathrm{d}}$, Sandra E. Black ${ }^{\mathrm{a}, \mathrm{b}, \mathrm{d}}$ \\ a Rotman Research Institute, Toronto, ON, Canada M6A 2E1 \\ b University of Toronto, ON, Canada \\ c Oxford Brookes University, Oxford, UK \\ d Sunnybrook Health Sciences Centre, Toronto, ON, Canada
}

\section{A R T I C L E I N F O}

\section{Article history:}

Received 13 December 2013

Received in revised form

6 February 2014

Accepted 8 February 2014

Available online 20 February 2014

\section{Keywords:}

Pathological recollection

Familiarity

Dementia

Déjà vu

Confabulation

Structural MRI

\begin{abstract}
A B S T R A C T
We report a single-case study of a female patient $(\mathrm{VL})$ who exhibited frequent episodes of erroneous recollections triggered by everyday events. Based on neuropsychological testing, VL was classified as suffering from mild to moderate dementia $(M M S E=18)$ and was given a diagnosis of probable Alzheimer's disease. Her memory functions were uniformly impaired but her verbal abilities were generally well preserved. A structural MRI scan showed extensive areas of gray matter atrophy particularly in frontal and medial-temporal (MTL) areas. Results of experimental recognition tests showed that VL had very high false alarm rates on tests using pictures, faces and auditory stimuli, but lower false alarm rates on verbal tests. We provide a speculative account of her erroneous recollections in terms of her MTL and frontal pathology. In outline, we suggest that owing to binding failures in MTL regions, VL's recognition processes were forced to rely on earlier than normal stages of analysis. Environmental features on a given recognition trial may have combined with fragments persisting from previous trials resulting in erroneous feelings of familiarity and of recollection that were not discounted or edited out, due to her impaired frontal processes.
\end{abstract}

Crown Copyright @ 2014 Published by Elsevier Ltd. All rights reserved.

\section{Introduction}

Déjà vu can be defined as an inappropriate feeling of familiarity, in which a situation, place or person feels strangely, yet erroneously, familiar. This sensation is commonplace but relatively fleeting, typically only experienced around once a year (Brown, 2003), and usually associated with a sense of temporary 'strangeness' by the person experiencing it. The incidence of déjà vu decreases with age in adulthood, but increases with education and income; it is often associated with fatigue or stress (Brown, 2003, 2004). The phenomenon is also associated with temporal lobe epilepsy-such patients sometimes experience a strong feeling of déjà vu in their preseizure aura. This observation suggests that the phenomenon reflects some disorder of neural processing, and this suggestion has been endorsed by a number of researchers. In overview, it appears that the experience of déjà vu is associated with overstimulation of regions in the medial temporal lobe (MTL), especially the hippocampus, parahippocampal cortex and rhinal cortices

\footnotetext{
* Corresponding author at: Rotman Research Institute, Baycrest Centre, 3560 Bathurst Street, Toronto, ON, Canada M6A 2E1. Tel: +1 416785 2500x3526; fax: + 14167852862 .

E-mail address: fcraik@research.baycrest.org (F.I.M. Craik).
}

(Bancaud, Brunet-Bourgin, Chauvel, \& Halgren, 1994; Bartolomei et al., 2004; Guedj, Aubert, McConigal, Mundler, \& Bartolomei, 2010; Spatt, 2002; Martin et al., 2012).

Moulin (2013), Moulin, Conway, Thompson, James, and Jones (2005), O'Connor, Lever, and Moulin (2010)) have described cases of persistent erroneous recollection in which the patients' behavior and reported experience resembled déjà vu, but appeared more vivid and specific. In these cases, patients claimed to remember having met people before, having seen TV programs already, and even of participating in a radio interview previously, when in fact none of these happenings had occurred. Moulin et al. (2005), O'Connor et al. (2010) refer to this condition as 'déjà vécu' (already lived) to distinguish such feelings of false recollection from the much vaguer feelings of false familiarity associated with déjà vu. Other cases of false recollection have been reported from time to time in the neuropsychological literature. Bancaud et al. (1994) evoked vivid 'memories' by administering deep brain electrode stimulation to temporal lobe epileptic patients in the areas of the hippocampus and amygdala. In a similar study, Bartolomei et al. (2004) gave cortical stimulation to epileptic patients as part of their pre-surgical evaluation, and found that memory-related experiences were reported most often after stimulation of the rhinal cortices-more often than after stimulation of the hippocampus or 
amygdala. Patients reported experiences of déjà vu most often during stimulation of entorhinal cortex, but experienced actual 'memories' most often during stimulation of the perirhinal cortex. Hamani et al. (2008) treated a patient with morbid obesity by deep brain stimulation in the ventral hypothalamus in an attempt to control his appetite. During this treatment, the patient unexpectedly reported the perception of a scene and individuals familiar to him from earlier in his life. Further investigation revealed that unilateral deep brain stimulation on either side led to a significant increase in activity in the hippocampus and parahippocampal gyrus regions, presumed to be due to stimulation of the nearby fornix. These recent reports are obviously similar to the earlier dramatic accounts of memories evoked by cortical stimulation by Penfield and Perot (1963).

Whatever the validity of the 'memories' evoked by electrical stimulation of MTL structures, the cases of déjà vécu reported by Moulin et al. (2005) strongly suggest that humans can experience feelings of recollection in the well-documented absence of a corresponding prior episode. It is also clear that these experiences of specific recollections differ markedly from the vague feelings of familiarity associated with déjà vu. The distinction between familiarity and recollection has been treated extensively in the cognitive psychology literature. Mandler (1980) discussed the difference between the two experiential states and pointed out that there are many instances in everyday life when a stimulus is familiar but cannot be attributed to its source in time and place. A typical occurrence is finding a face intensely familiar when the person is encountered in an unusual context, yet being unable to say why the person seems so familiar-'the butcher on the bus' phenomenon (Mandler, 1980). Tulving (1985) drew a similar distinction between simply 'knowing' that an event has occurred previously and 'remembering' something specific about its prior occurrence; he suggested that these different mental states may have different brain correlates (Tulving, 1973, 1985). Further progress in understanding and specifying the differences between the two states was enabled by Jacoby's (1991) process dissociation procedure which provided a means to measure and separate familiarity from recollection. Reviews of the distinction have been provided by Gardiner and Richardson-Klavehn (2000) and by Yonelinas (2002).

Evidence for different brain mechanisms corresponding to these different experiential states has been accruing over the past 10-15 yr. In their original observations, Penfield and Perot (1963) found that memory-like experiences in their epileptic patients were induced by electrical stimulation of the lateral temporal neocortex, especially the superior temporal gyrus. Following up on these findings, Bancaud et al. (1994) also observed patients with temporal lobe epilepsy, and confirmed that the experience of déjà $\mathrm{vu}$ or memory hallucinations - induced either via electrical stimulation or by a spontaneous seizure - involved a neural network consisting of the anterior hippocampus, amygdala and superior temporal gyrus. Spatt (2002) suggested that the parahippocampal gyrus and its neocortical connections were responsible for the feelings of familiarity that normally accompany conscious recollection. In cases of déjà $\mathrm{vu}$, he proposed that current perception of a novel scene co-exists with inappropriate activation of the parahippocampal cortex, yielding an intense feeling of familiarity. Spatt's hypothesis followed the work of Aggleton and Brown (1999) who presented evidence for hippocampal involvement in the conscious recollection $(R)$ of contextual elements, as opposed to feelings of familiarity $(F)$ which were generated by a different circuit including the parahippocampal cortex. Rugg and Yonelinas (2003) reviewed evidence for the $F / R$ distinction, and concluded that the findings are consistent with the notion that hippocampal activation is a correlate of recollection whereas familiarity depends on other structures in the anterior MTL region. A more specific suggestion was made by Barbeau et al. (2005) who implanted electrodes as part of the presurgical procedure in the right temporal lobe of a patient suffering from right temporal lobe epilepsy. Electrical stimulation delivered through contacts located just lateral to the perirhinal cortex evoked vivid visual perceptions of familiar scenes, although these 'memories' were apparently unrelated to any specific past event. These vivid mental experiences were associated with synchronized electrical activity recorded from medial-temporal structures and between these structures and primary visual areas. The authors suggest that these various areas work in concert using the mechanism of synchronization of neural firing patterns in the theta range.

Based on these findings and suggestions, Diana, Yonelinas, and Ranganath (2007) proposed a three-component model in which the perirhinal cortex represents specific item information, the parahippocampal cortex encodes and retrieves contextual information, and the hippocampus supports recollection by associating item and contextual information. At the time of retrieval, item familiarity is signaled by activity in perirhinal cortex and hippocampus, leading to reactivation of the associated contextual information in the parahippocampal cortex. Thus, familiarity and recollection are associated with different regions in the MTL. These suggestions are entirely compatible with observations on clinical cases reported by Bowles et al. (2007, 2010). In the first study, this group reported the case of an individual who had undergone surgical resection of left-temporal lobe structures for the treatment of epilepsy. The resection included a large portion of perirhinal cortex but spared the hipppocampus. In line with the three-component model, this patient exhibited impaired familiarity with preserved recollection (Bowles et al., 2007). In a later article, the group reported findings from 10 individuals whose surgical resections involved the hippocampus but spared the perirhinal cortex; in this case comparison with healthy controls revealed impaired recollection but preserved familiarity (Bowles et al., 2010).

Theoretical accounts of the phenomenon are discussed again in the Discussion, but are briefly outlined here. Spatt's (2002) account of déjà vu has been described already. Moulin et al. (2005) suggested that erroneous feelings of recollection were attributable to over-active or ungated recollective processes triggered by events that attracted the patient's attention. That is, déjà vécu experiences reflect inappropriate activation of the neural structures that are normally concerned with signaling recollection. O'Connor et al. (2010) developed this idea by suggesting that the phenomenon is typically triggered by novelty, and is associated with inappropriate interpretation of output signals from the hippocampus by frontal regions. Specifically, they suggest that prefrontal neurons occasionally fire in response to output from the hippocampus during a phase of oscillatory activity in the theta frequency band that is normally associated with hippocampal retrieval, even when the information being processed is actually associated with encoding. In a sense, then, encoding is occasionally interpreted and signaled as retrieval, thereby giving rise to erroneous feelings of recollection. In a later paper, Moulin (2013) extended his earlier views and suggested that some novel events give rise to erroneous feelings of intense familiarity in such patients. In response to this feeling, the cognitive system 'assumes' that the familiarity must be due to some prior experience and so manufactures a scenario to fit the case. That is, erroneous recollection is attributable to what Moulin terms 'recollective confabulation.' Many of the 11 cases described by Moulin (2013) have suspected frontal lobe damage, so the suggestion of confabulation is reasonable. His notion also fits well with nonpathological examples in cognitive experiments in which induced familiarity is wrongly attributed to a prior occurrence (Jacoby \& 
Whitehouse, 1989) or to the erroneously perceived 'fame' of an arbitrary name (Dywan \& Jacoby, 1990).

We present here an account of another patient whose experiences and behaviors closely match those of the patients described by Moulin (2013). After giving a general description of the patient and her memory problems, we describe her clinical assessment, findings from a structural brain scan, and the results of some experimental cognitive tests. We conclude by suggesting possible neural correlates of her faulty memory experiences in light of her atypical behavioral results and the specific pattern of brain lesions revealed by scanning.

\subsection{Case report}

VL is now deceased, but at the time of initial testing was a welleducated woman ( $13 \mathrm{yr}$ of formal education) aged $81 \mathrm{yr}$. The investigations reported in this article took place over two years. She was widowed and lived in a home for senior citizens in Toronto, Canada. She was in good physical health for her age. Her daughter routinely drove VL to a local shopping center where, on the way, she might comment on a man standing at a bus stop, observing that it was surprising that he was always there at the same time and in the same place. As well, in the shopping center she might draw the daughter's attention to a woman shopper and comment that she always seemed to be there when they visited, although neither the man at the bus stop nor the woman shopper had been encountered previously. Observations of this type occurred on virtually every outing. Her daughter also took VL for day trips from time to time, and noted that her mother would often remark casually on aspects of the scene that caught her attention, as if she was remembering it from a prior occasion, although the daughter knew clearly that the mother had never visited that location before. For example, while passing through a small town VL noticed a shabbily dressed man standing on a corner and exclaimed "You'd think that man would have other things to do - he's always standing on that corner!" Other similar incidents involved a man weaving through traffic on a bicycle ("He's here every time we come to this place!") and a young woman who startled VL by jogging past her on the sidewalk ("She's always doing that - you'd think she would be more careful!"). Such remarks seemed to be prompted by incidents that caught her attention, and had often some negative emotional connotation ("These people are always leaving their garbage strewn around their front yard!"), and this appears to be typical of the cases that have been described to date (Moulin, 2013). Additionally, VL would claim that she had been shown holiday photographs before, when in fact she had not seen them. She also complained that she had seen (new) TV programs already. Initially, her daughter would tell her that the programs (frequently news broadcasts) could not possibly be repeats, but stopped making this comment because her mother became annoyed and clearly did not believe her. This paramnesia extended beyond people, buildings and broadcasts. For example, she once expressed annoyance that she had received a second letter from her grand-daughter in California that was exactly the same as a letter the granddaughter had sent once before. VL felt insulted and expressed anger that her grand-daughter did not care enough to be bothered to write a new letter and simply re-sent the previous one. In fact, no letter had ever been sent, so clearly confabulation was one of VL's symptoms. Similar episodes had been occurring since VL was in her mid- to late 70s.

These remarks and many similar ones were made without surprise or comment. VL clearly felt that she was re-experiencing day-to-day events, and was recollecting the first occasion on which they had occurred. Because of such incidents, and other signs of mental difficulties including early signs of dementia (e.g., difficulty in keeping track of possessions, forgetting recent events, forgetting previously familiar geographic routes), VL's daughter took her to a neurology clinic where she was given a number of clinical tests, described in the next section. At the same time, we brought her into a cognitive psychology lab and administered various experimental tests to assess her memory abilities. These tests were designed to simulate real-world situations that provoked VL's excessive experiences of false familiarity and recollection. On the basis of her case history we hypothesized that VL would show an abnormally high false alarm rate in recognition memory tasks.

\subsection{Clinical assessments}

When she was aged 83, VL was referred to Sunnybrook Health Sciences Centre in Toronto to be assessed for possible Alzheimer's disease. Her cognitive abilities were assessed on a standardized battery of neuropsychological tests that have been used in the Sunnybrook Dementia Study for the last $25 \mathrm{yr}$. She also had a standardized MRI scan that permitted volumetric brain analysis (reported below). On the basis of her performance on these various tests, VL was diagnosed as suffering from probable Alzheimer's disease.

\subsubsection{Neuropsychological examination}

The results of the neuropsychological tests are shown in Table 1 . VL clearly was an individual of above average intelligence, who at the time of examination was suffering from a mild to moderate dementia. The first point follows from her estimated full-scale IQ (115) calculated from the New Adult Reading Test (NART) which gives a measure of pre-morbid intelligence; also from her WAIS Vocabulary score of 56/60 which is in the high normal range. These measures of crystallized intelligence contrast with scores from tests containing items that have been less rehearsed, such as the Mini-Mental Status Exam (MMSE) and the Mattis Dementia Rating Scale (MDRS). Both of these latter tests showed evidence of intellectual deterioration, in contrast to her performance on well-rehearsed information. The profile of results indicates that several abilities were more severely compromised than others, with many abilities remaining within normal limits for her age. Simple attention (MDRS Attention, Digit Span Backwards) and speed of processing (Digit Symbol, phonemic fluency) were intact. There was no evidence of significant visual-spatial disturbance, with Judgment of Line Orientation and Benton Facial Recognition scores within normal limits. All aspects of conversational speech were within normal limits on the Western Aphasia Battery (WAB). VL had low normal performance on the 64-card version of the Wisconsin Card Sorting Task (WCST), but most importantly, she did not exhibit an unusually large number of perseverative errors.

In contrast, there was evidence of dysfunction in several domains. On the 30-word Boston Naming Test (BNT), she was impaired on naming pictured objects (perhaps reflecting the greater difficulty level of the BNT picture list compared to the WAB objects). There was a notable difference between performance on semantic fluency (less than 10th percentile) compared with phonemic fluency (FAS-scaled score of 15, above normal), suggesting more involvement of temporal than frontal regions (Glisky, Polster, \& Routhieaux, 1995). There was evidence of mild ideomotor apraxia on the WAB apraxia subtest. However, her most obvious impairment occurred in verbal and nonverbal memory, as evidenced by performance on the California Verbal Learning Test (CVLT), the Visual Reproduction task from the WMS-R battery, and the single dysfunction on the relatively simple recognition memory subtest of the MDRS. 
Table 1

Neuropsychological test results for VL at time of MRI.

\begin{tabular}{|c|c|c|}
\hline Test & Score & Performance level \\
\hline \multicolumn{3}{|l|}{ General function: } \\
\hline Mini-Mental Status Exam (MMSE) & $18 / 30$ & Mild to moderate dementia \\
\hline Mattis Dementia Rating Scale (DRS) total & $125 / 144$ & Low normal $\left(\mathrm{ss}=6^{\mathrm{a}}\right)$ \\
\hline Attention & $36 / 37$ & Normal to high normal $(\mathrm{ss}=12)$ \\
\hline Initiation & $36 / 37$ & Normal $(\mathrm{ss}=11)$ \\
\hline Construct & $6 / 6$ & Normal $(\mathrm{ss}=10)$ \\
\hline Concept & $37 / 39$ & Normal $(\mathrm{ss}=11)$ \\
\hline Memory & $10 / 25$ & Impaired $(\mathrm{ss}=2)$ \\
\hline NART full-scale IQ & 115.32 & Above average (normal =100) \\
\hline \multicolumn{3}{|l|}{ Language tests: } \\
\hline Boston naming & $11 / 30$ & Impaired $\left(z\right.$ score $\left.=-3.92^{\mathrm{b}}\right)$ \\
\hline Semantic fluency & 9 & Borderline ( $<10$ th percentile) \\
\hline \multicolumn{3}{|l|}{ Visuospatial ability: } \\
\hline Rey copy & $21.5 / 36$ & Low normal (25th percentile) \\
\hline Line orientation & $24 / 30$ & Normal $(\mathrm{ss}=12)$ \\
\hline Line orientation age and sex corrected & $29 / 30$ & High normal (86th percentile) \\
\hline \multicolumn{3}{|l|}{ Memory: } \\
\hline \multicolumn{3}{|l|}{ CVLT recall measures: } \\
\hline Acquisition ( $T$-score) & 38 & Low average \\
\hline Short Delay ( $z$ score) & -3 & Impaired \\
\hline Long delay ( $z$ score) & -4 & Impaired \\
\hline Error: perseverations & 0 & High normal \\
\hline WMS-R immediate visual reproduction & $8 / 41$ & Impaired (2nd percentile) \\
\hline WMS-R delayed visual reproduction & $0 / 41$ & Impaired (4th percentile) \\
\hline WMS-R digit span forward & $6 / 12$ & Low normal (22nd percentile) \\
\hline WMS-R digit span backward & $10 / 12$ & High normal (92nd percentile) \\
\hline \multicolumn{3}{|l|}{ Executive function: } \\
\hline FAS fluency (age and education corrected) & 54 & Above average fluency ( $s s=15$ ) \\
\hline \multicolumn{3}{|l|}{ Wisconsin card sorting test (WCST): } \\
\hline Categories & $3 / 6$ & Normal $(z$ score $=-0.34)$ \\
\hline Total number correct & 51 & Low normal $(z$ score $=-1.17)$ \\
\hline Total number of errors & 13 & Low normal $(z$ score $=-1.38)$ \\
\hline Perseverations to preceding category & 9 & \\
\hline Perseverations to preceding response & 0 & \\
\hline \multicolumn{3}{|l|}{ Additional tests: } \\
\hline Benton facial recognition (age and education corrected) & $49 / 54$ & Normal \\
\hline Western Aphasia Battery (WAB) & $96.2 / 100$ & Normal \\
\hline Spontaneous speech & $19 / 20$ & \\
\hline Fluency & $10 / 10$ & Normal \\
\hline Content & $9 / 10$ & Normal \\
\hline Comprehension & $10 / 10$ & Normal \\
\hline Repetition & $9.6 / 10$ & Normal \\
\hline Naming & $9.5 / 10$ & Normal \\
\hline Reading Quotient & $100 / 100$ & Normal \\
\hline Writing Quotient & $96 / 100$ & Normal \\
\hline WAB apraxia & $48 / 60$ & Normal \\
\hline WAIS vocabulary & $56 / 60$ & High normal $(\mathrm{ss}=12)$ \\
\hline
\end{tabular}

a Scaled scores (ss) normal $=7$ to 13 .

${ }^{\mathrm{b}} z$-Scores normal $=-1.5$ to $+1.5=5$ th-91st percentile).

This profile of results comparing VL's relative strengths and weaknesses in cognitive functioning suggests that, at the time of assessment, maximum pathology was within the temporal lobes. In other cortical domains, performance was either within normal limits or was even superior for her age; for example, tests of parietal function (JLO; normal comprehension on the WAB), and frontal function (phonemic fluency; WCST). Results from a structural MRI scan were also available and are described in the next section.

\section{Structural neuroimaging analysis}

\subsection{Control participants}

Comparisons for the neuroimaging analysis were made with two separate groups of control participants who were men and women $78 \mathrm{yr}$ of age and older. The first comparison was a whole brain analysis, contrasting VL's measurements with a group of 15 healthy normal control participants who had no history of neurological or psychiatric illness (mean age $=81.9 \mathrm{yr}, \mathrm{SD}=2.9 \mathrm{yr}$; mean education $=15.8 \mathrm{yr}, \mathrm{SD}=3.1 \mathrm{yr}$; $\mathrm{MMSE}=28.7, \mathrm{SD}=1.0$ ). This analysis comprised a detailed quantification of regional brain tissue volumes using previously published methods (Dade et al., 2004; Kovacevic et al., 2002). The second set of comparisons involved a detailed hand-traced analysis of MTL structures. VL's measurements were compared to those from a further group of 6 normal control participants (mean age $=76.7 \mathrm{yr}, \mathrm{SD}=2.1$; mean education $=13.3 \mathrm{yr}$, $\mathrm{SD}=2.4 \mathrm{yr}$; $\mathrm{MMSE}=28.7, \mathrm{SD}=1.5$ ). Controls were recruited from the Sunnybrook Dementia Study for which they gave informed, written consent. This study was approved by the Research Ethics Board of Sunnybrook Health Sciences Centre, an academic healthcare institution fully affiliated with the University of Toronto.

In the analyses below, VL was compared to each respective control group using Crawford's modified t-tests designed to compare a single case to a control sample (Crawford, Garthwaite, \& Howell, 2009). Given our directional hypotheses (i.e., VL would have reduced gray matter volumes), these tests were one-tailed. 


\subsection{MRI acquisition}

All subjects were imaged using a 1.5 T-MR system (Signa, General Electric) using a standard protocol including an axial
T1-weighted 3D volume acquisition yielding 124 slices (TR/TE of $35 / 5 \mathrm{~ms}$, flip angle of 35 degrees, $1.0 \mathrm{NEX}$, FOV of $22 \mathrm{~cm}$, and slice thickness 1.2 to $1.4 \mathrm{~mm}$ ). Proton density and T2-weighted images with a slice thickness of $3 \mathrm{~mm}$ were obtained using an interleaved

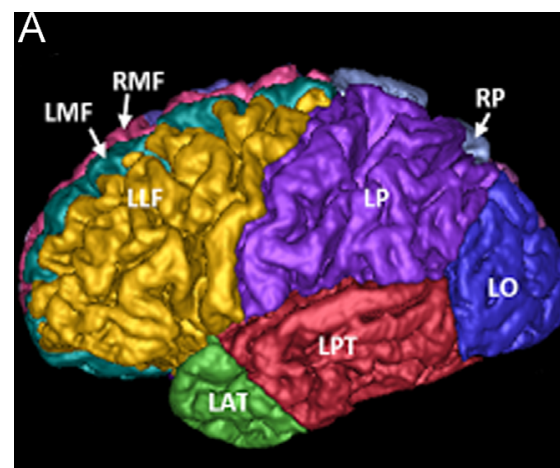

C

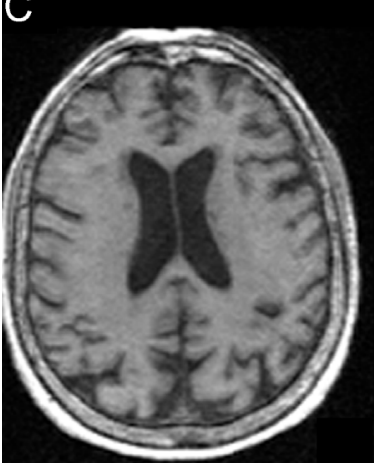

$\mathrm{D}$

F

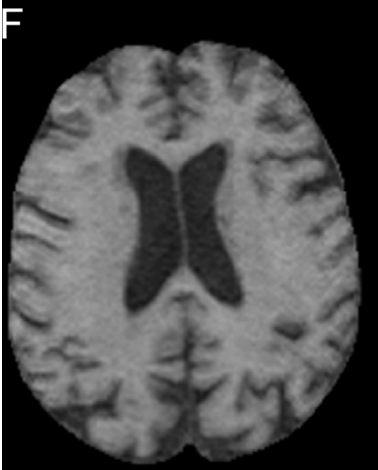

|

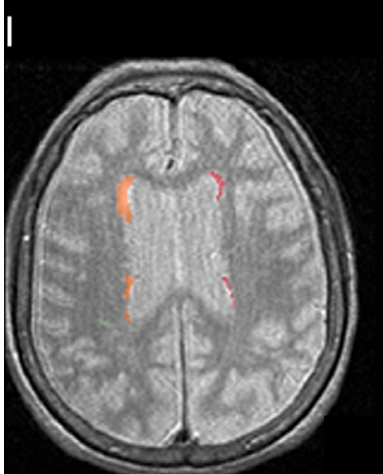

?

B

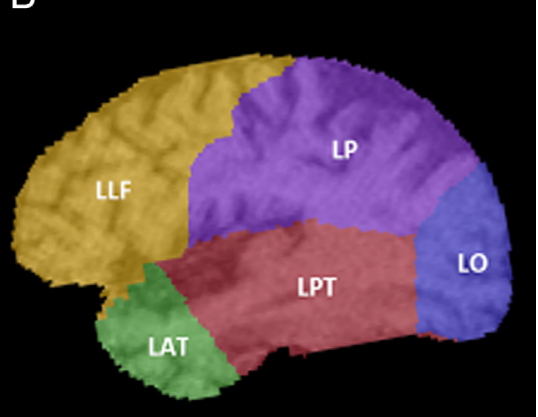

$E$

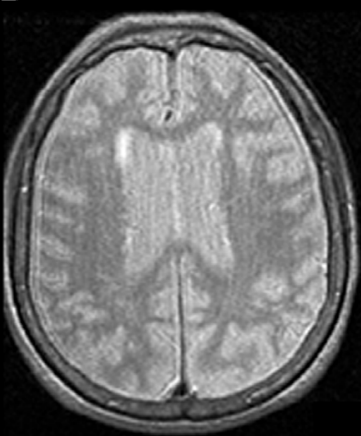

G

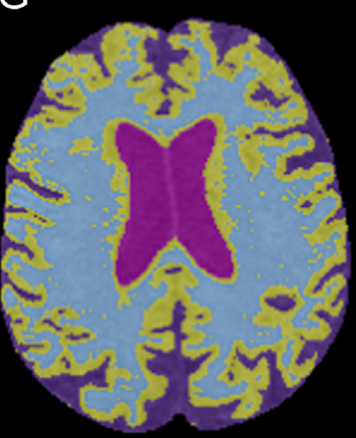

$\mathrm{J}$

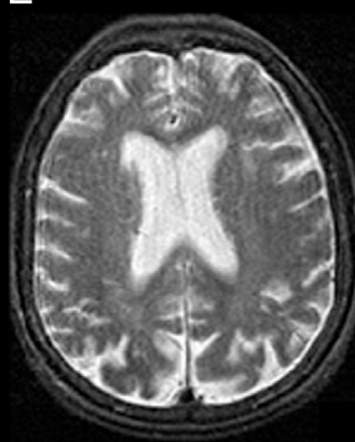

$\mathrm{H}$

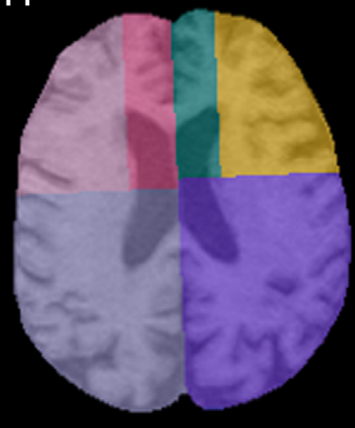

K
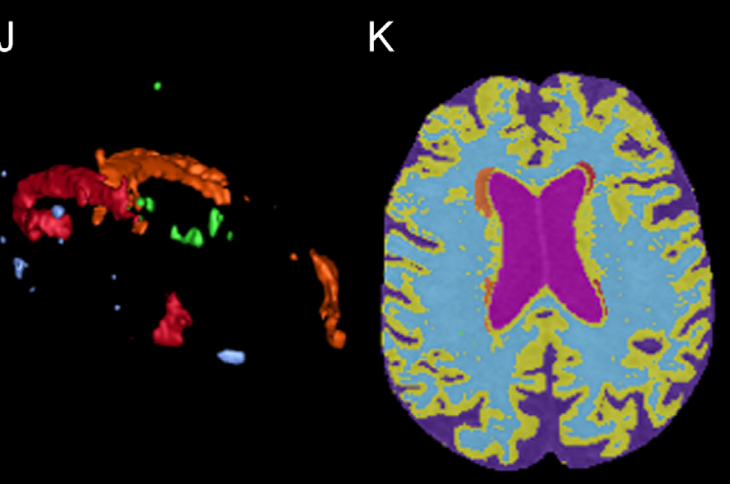

Fig. 1. (A)-(B) Semi-automatic brain region extraction (SABRE) parcellated brain with colors representing different brain regions: $L M F=l$ left $(L)$ medial frontal, $L L F=L$ lateral frontal, $\mathrm{LAT}=\mathrm{L}$ anterior temporal, $\mathrm{LPT}=\mathrm{L}$ posterior temporal, $\mathrm{LO}=\mathrm{L}$ occipital, $\mathrm{LP}=\mathrm{L}$ parietal, $\mathrm{RP}=$ right $(\mathrm{R})$ parietal, $\mathrm{RMF}=\mathrm{R}$ medial frontal; (C) $3 \mathrm{D}$ T1-weighted $\mathrm{MRI}$, to which a proton density (D) and T2-weighted (E) image are co-registered, (F) Masked T1 using total intracranial vault extraction from co-registered PD/T2, (G) tissue segmentation from masked T1: grey matter (yellow), white matter (blue), ventricular (v) CSF (fuchsia), sulcal (s) CSF (purple), (H) example axial slice showing regional SABRE parcellation, (I) lesion mask overlaid on proton density: Left periventricular (pv) subcortical hyperintensity ( $\mathrm{SH}$ ) (red), right pvSH (orange), (J) 3-D rendered lesion mask, left pvSH (red) and deep white (dw) SH (blue), right pvSH (orange) and dwSH (green), (K) combined tissue and lesion segmentation giving 8 separate tissue classes. (For interpretation of the references to color in this figure legend, the reader is referred to the web version of this article.) 
sequence (TR/TE of 3000/30, $80 \mathrm{~ms}, 0.5 \mathrm{NEX}$, and FOV of $22 \mathrm{~cm}$ ), permitting reliable quantification of subdural cerebrospinal fluid (SCF), and white matter hyperintensities (Kovacevic et al., 2002).

\subsection{Whole brain analysis}

In brief, image processing for analyses of whole brain volumetrics included skull-stripping, tissue segmentation, regional brain parcellation, and classification of hyperintensities into periventricular, deep white and lacunar infarcts, in accordance with our in-house pipelines (Dade et al., 2004; Kovacevic et al., 2002; Levy-Cooperman, Ramirez, Lobaugh, \& Black, 2008; Ramirez et al., 2011). More information on this pipeline can also be found at $<$ sabre.brainlab.ca $>$. These methods were applied to the case study patient (VL) and to the first set of 15 control participants to delineate 9 regions of interest (Fig. 1A and B).

Reliable dissociation of brain and associated cerebrospinal fluid from the head and skull was achieved by applying the procedure "Brain Sizer", which utilizes proton density (PD) and T2-weighted images co-registered to the T1 (Fig. 1C and E) in order to obtain both an accurate input image for tissue segmentation and allow for a reliable measure of the total intracranial vault for head-size correction (Kovacevic et al., 2002; Ramirez et al., 2011). This procedure is performed individually for each subject with a trained rater performing manual edits to remove minor misclassified voxels (such as the eyes, cranial nerves or dura), with high reliability (see Ramirez, Scott, \& Black, 2013 for a full inter-rater reliability analysis for this step as well as the others to follow). This skull-stripped T1 image (Fig. 1F) is then automatically segmented into gray matter, white matter, and cerebrospinal fluid (CSF) using a multi-feature local and global histogram approach, after which the ventricular CSF is manually relabeled (Fig. 1G) to differentiate it from sulcal CSF for separate quantification (Kovacevic et al., 2002; Ramirez et al., 2011).

Regional parcellation (termed SABRE-semi-automatic brain region extraction) is achieved through a series of semi-automated steps including anterior-commissure posterior-commissure alignment of the T1 image to create a unique subject-specific Talairachlike grid, and identification of 15 landmarks and tracings including the left and right Sylvian fissure, central sulcus, and parietooccipital sulcus. These landmarks and tracings are then automatically combined with the Talaraich-like grid to generate a parcellation mask (Fig. 1A, B and $\mathrm{H}$ ) that can be applied to the tissue segmentation to generate 26 regions of interest (13 per hemisphere) for each of the tissue classes (Dade et al., 2004; Ramirez et al., 2011). Further segmentation of white matter hyperintensity (WMH) tissue classes is achieved through a semi-automated technique called "Lesion Explorer," which utilizes a tri-feature algorithm based on PD/T2 and T1 to quantify hyperintensities into 4 sub-classes (Fig. 1I and J) including both periventricular and deep hyperintensities and CSF-filled "black holes", with minor manual editing by a highly trained rater to remove false positives (Ramirez et al., 2011). Together these pipelines yield individualized subjectspecific volumetric data for 8 tissue classes (Fig. $1 \mathrm{~K}$ ) for up to 26 regions of interest, which can be further combined to create summary volumetrics for various total lobes (medial/lateral frontal, total parietal, temporal, etc.). For the purposes of this analysis, 3 lateral frontal, 3 medial frontal and 2 parietal sub-regions were further combined to yield total lateral frontal, total medial frontal and total parietal regions, respectively.

Results from these analyses are shown in Fig. 2. Crawford's $t$-tests to compare VL to controls revealed that VL had significantly reduced gray matter in the left and right frontal lobes as a whole $[t(15)=-1.82, p<0.05$ and $t(15)=-1.92, p<0.05$ respectively $]$, more specifically in lateral frontal regions [left: $t(15)=-1.86, p<$ 0.05 , right: $t(15)=-2.02, p<0.05$ ]. VL also showed significantly
A

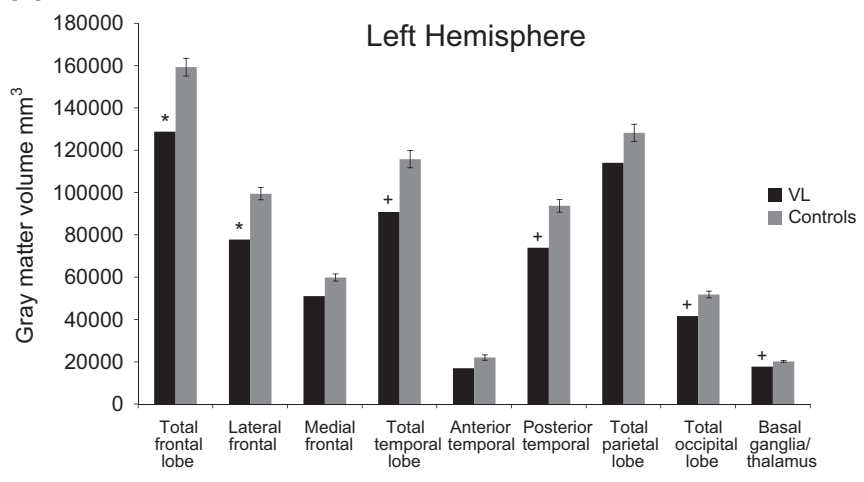

B

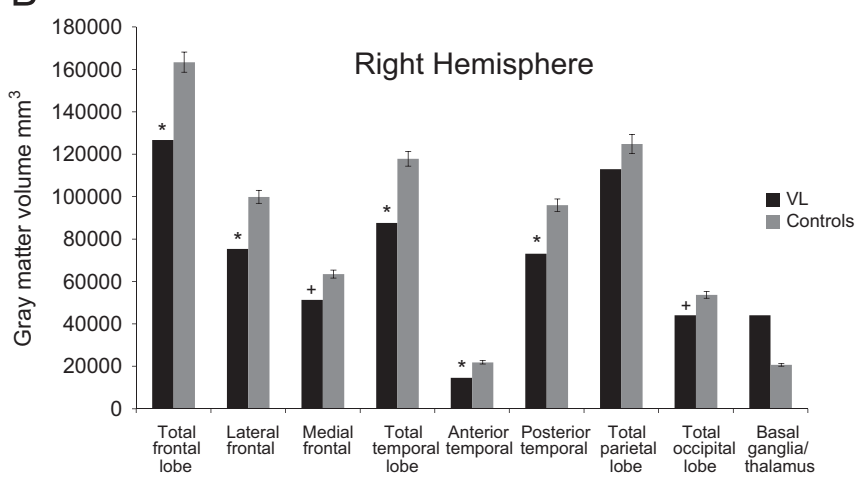

Fig. 2. Comparison of automatically-obtained brain gray matter volumes in VL and normal controls for the left (A) and right (B) hemispheres. Error bars reflect SEM. ${ }^{*} p<.05,{ }^{+} p<.10$.

reduced gray matter in the right temporal lobe measured as a whole $[t(15)=-2.20, p<0.05]$, which included significant damage to both the right anterior temporal lobe $[t(15)=-2.18, p<0.05]$ and the right posterior temporal lobe $[t(15)=-1.94, p<0.05]$. She also showed marginally reduced gray matter in the following regions: left temporal lobe as a whole [ $t(15)=-1.54, p=0.07]$, left posterior temporal lobe $[t(15)=-1.66, p=0.06]$, left and right occipital lobe $[t(15)=-1.63, p=0.06$ and $t(15)=-1.50 p=0.08$, respectively], left basal ganglia/thalamus $[t(15)=-1.40, p=0.09]$, right medial frontal lobe $[t(15)=-1.62, p=0.06]$.

\subsection{Medial temporal lobe volumetry}

We additionally performed a more detailed examination of the MTL in VL and the second set of normal control participants using manual tracing on T1-weighted images according to the methods of Callen, Black, Gao, Caldwell, and Szalai (2001) for three MTL regions: the hippocampus, parahippocampal cortex and amygdala-entorhinal-perirhinal (AEP) complex (Fig. 3). The volumes were corrected for total intracranial capacity. Fig. 3A and D shows the tracing methods for these structures, as well as comparisons between 6 healthy controls and VL. Crawford's t-tests to compare VL to controls revealed significantly reduced volume bilaterally in the hippocampus [left: $t(5)=-4.58, p<0.01$, right: $t(5)=-3.70$, $p<0.01$ ] and parahippocampal cortex [left: $t(5) 6.94, p<0.01$, right: $t(5)=6.67, p<0.01$ ]. The measure of the AEP appeared to be within normal limits [left: $t(5)=0.36, p=.73$, right: $t(5)=0.63$, $p=0.55]$. Due to low scan quality in VL which caused poor gray and white matter differentiation in the anterior medial temporal lobe, we were unfortunately not able to analyze the perirhinal cortex separately from the amygdala and entorhinal cortex. However, there is reason to believe that perirhinal cortex was significantly atrophied in VL. First, visual assessment of her lesion 

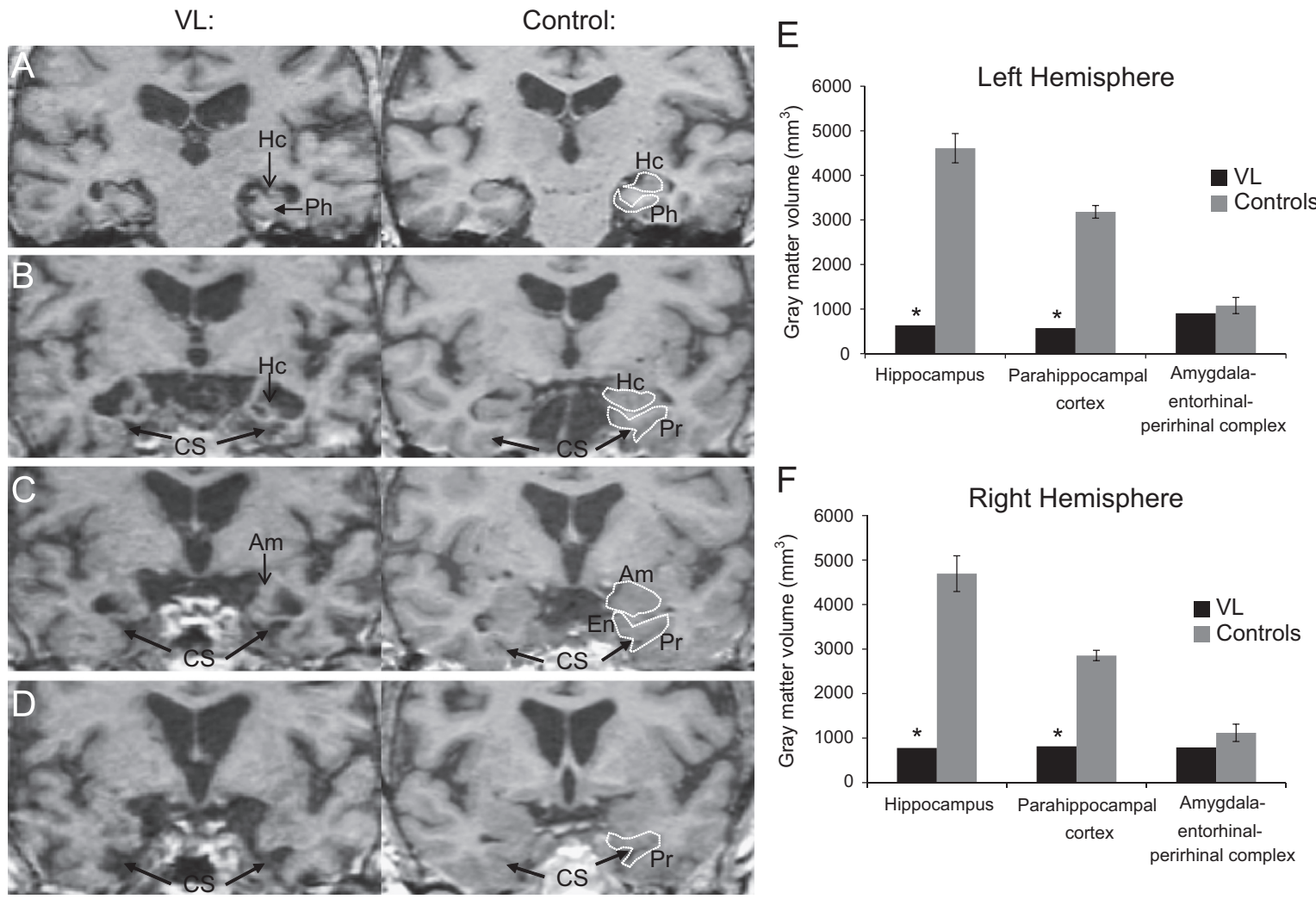

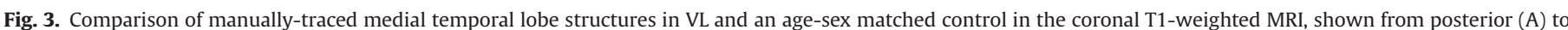

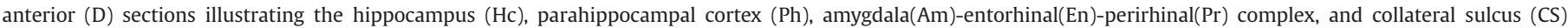

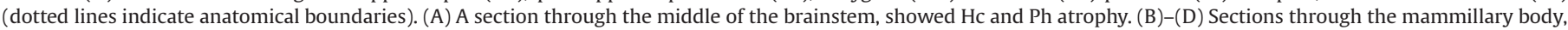

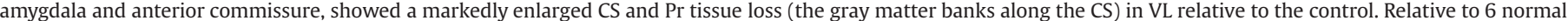
controls, VL showed atrophy in the hippocampus, and parahippocampal gyrus in both the left (E) and right (F) hemispheres. Error bars reflect SEM. * $p<.05$.

demonstrated marked enlargement of the collateral sulcus as well as perirhinal shrinkage (Fig. 3B and D). In addition, the parahippocampal cortex region defined by the volumetric method used in the current study (Callen et al., 2001) included part of the posterior portion of the perirhinal cortex. As such, the documented tissue loss in parahippocampal cortex (Fig. $3 \mathrm{E}$ and F) can be partially attributed to perirhinal cortex atrophy.

Thus, in summary, VL's tissue losses were primarily in frontal and MTL regions, with damage in the latter being mainly in hippocampus, parahippocampal cortex and, to a lesser degree, likely also perirhinal cortex.

\section{Experimental tasks}

In order to assess VL's memory problems in greater detail, we devised a series of experimental recognition memory tasks that utilized a variety of different materials-pictures, sounds, words, sentences and faces. Given that these tests were designed to explore VL's memory strengths and weaknesses across a range of materials, the tests were constructed to suit the materials, and participants' abilities with the materials, rather than to be directly comparable to each other. The tests for pictures and sounds did follow the same pattern - 135 trials with 23 repeated-items at lags of 20 intervening items - but the tests for faces, sentences and single words added the feature of variable lags for repeated items. Additionally, the sentence test explored the variable of concrete versus abstract sentences, and the word test examined the effects of testing the repeated words in the same or a different mode of presentation (visual or auditory) from their initial appearance. Again, the main point of these various tests was to assess VL's recognition memory in as many different ways as possible.

On the basis of her case history, we expected to find abnormally high false alarm rates on these tests. For comparison purposes we also tested a group of 12 older adults whose mean age was $77.6 \mathrm{yr}$ $(\mathrm{SD}=6.56)$, and who had a mean of $14.5 \mathrm{yr}$ of education $(\mathrm{SD}=3.33)$. At the time of testing, VL was aged 81. Results from these tests are shown in Table 2. We again compared VL's performance to that of controls using Crawford's modified $t$-tests (Crawford et al., 2009).

\subsection{Test 1. Picture recognition memory}

The visual version of this test was constructed from 112 pictures taken from magazines that VL was unlikely to have seen, mostly British magazines. The pictures were largely neutral scenes, but included a few positive and negative emotional pictures. Of the 112 pictures presented, 23 were repeated at lags of 20 intervening items-thus the total set consisted of 135 items. The pictures were pasted into a loose-leaf binder and participants were shown the successive pictures at their own pace. For each item, participants first decided whether or not they had seen that item before in the series; they answered yes or no, and also gave a confidence rating in their judgment in which $3=$ certain, $2=$ fairly sure and $1=$ possibly. Second, they judged whether they had ever seen that picture before (e.g., in a magazine); again the judgment was yes or no plus a confidence rating.

In this test, the hit rate (the proportion of 'yes' responses to repeated pictures) for the control participants was 0.97 compared 
Table 2

Means (and SDs) on the five recognition memory tests for normal controls and VL.

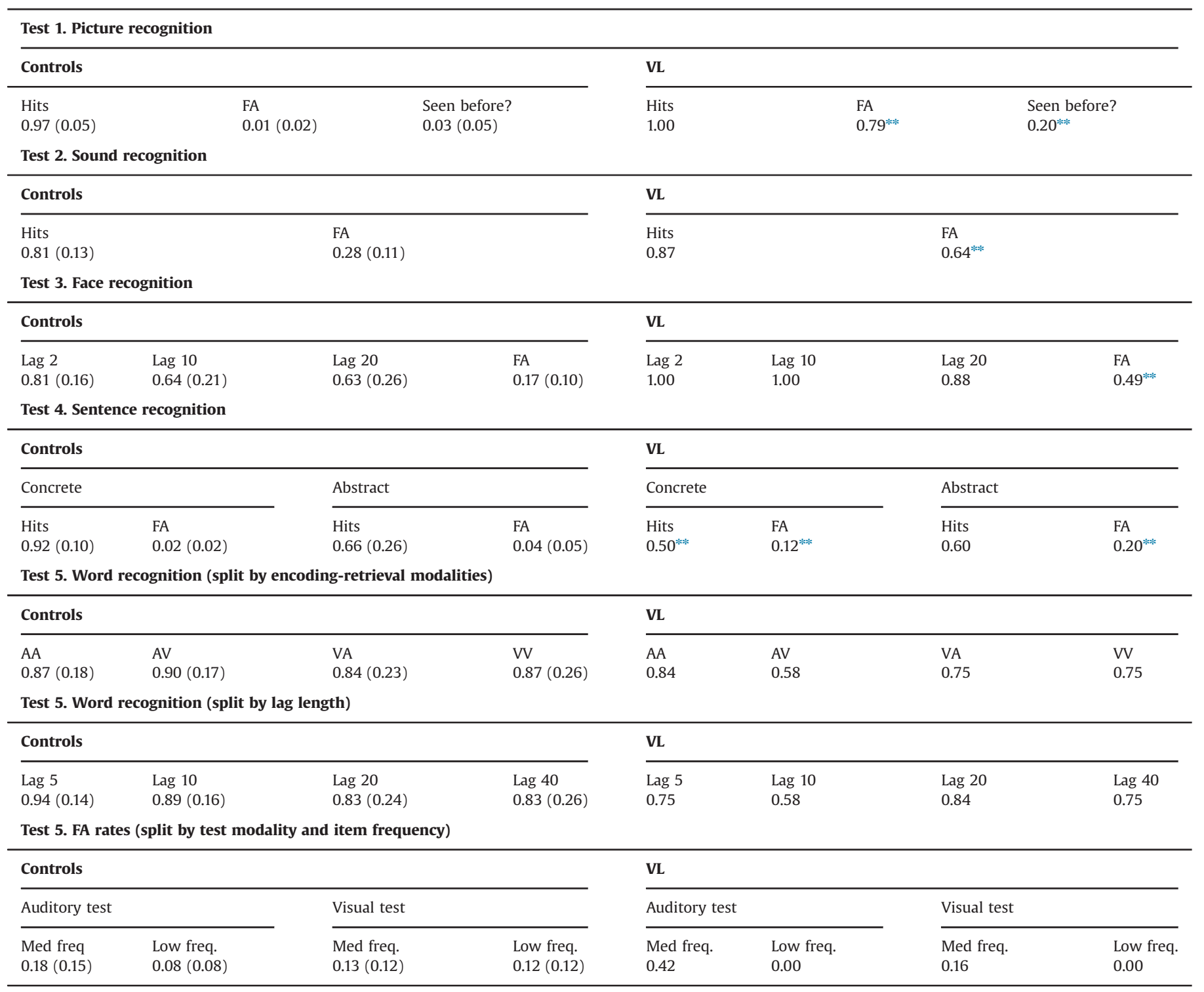

** $p<0.01$ for the comparison of VL versus normal controls. FA=false alarms. For Test $5, A=$ auditory, $V=$ visual; 1 st and 2 nd letters refer to the encoding and retrieval modalities respectively.

to VL's hit rate of 1.00-a non-significant difference, $t(11)<1.0$. The mean confidence rating for hits was 2.98 for the controls and 2.61 for VL, and this difference was significant by Crawford's $t$-test, $t(11)=11.94, p<.001$. The false alarm rate (FA, the proportion of 'yes' responses to pictures presented for the first time) for controls was 0.01 compared to 0.79 for VL. This difference was highly significant, $t(11)=37.47, p<.001$. Additionally, VL's false alarm rate increased in the course of the series; rates for successive quartiles of the test were $0.56,0.89,0.88$ and 0.88 for first, second, third and final quartiles respectively. Confidence levels in false alarms (thus erroneously high confidence in errors) also showed a tendency to increase as the series went on; the ratings were 1.44, 1.80, 2.00 and 1.91 for first, second, third and final quartiles respectively, with a mean confidence rating of 1.81 . It was not possible to carry out a similar quartile analysis of false alarms in the control group as they made very few errors-only 14 false alarms out of a possible 1344 opportunities. The mean confidence rating for controls' FAs was 1.93. The proportions of yes responses to the question 'have you ever seen the picture before outside the testing session?' were 0.03 for controls and 0.20 for $\operatorname{VL}[t(11)=3.27, p<.01]$. Thus, the prediction that VL would show an atypically high false alarm rate was strongly confirmed.

\subsection{Test 2. Sound recognition memory}

In order to assess whether VL's memory impairment was primarily or exclusively visual in nature, we designed an analogous test using environmental sounds taken from a CD of sound effects for theater or radio productions. Again, 112 brief sound segments (2-15 s in duration) were played to participants at their own pace, and 23 of these were repeated at lags of 20 intervening items. After listening to each sound, participants judged whether the sound was new or a repeat and gave a confidence rating in their response. The results showed that the controls had a hit rate of 0.81 compared to VL's rate of $0.87(t<1)$. The mean confidence rating for hits was 2.55 for controls and 2.00 for VL, a nonsignificant difference, $t(11)=1.43, p>.05$. The FA rate for controls was 0.28 compared with 0.64 for $\operatorname{VL}[t(11)=3.14, p<0.01]$. As with picture recognition, VL's false alarm rate increased across the 135 trials; rates for successive quartiles of the test were $0.32,0.71,0.79$ 
and 0.71 for first to fourth quartiles, respectively. Her confidence levels in these false alarm responses also increased from first to fourth quartiles; mean confidence ratings were 1.33, 1.60, 1.59 and 1.90 respectively, with an overall mean of 1.65 . The corresponding FA rates for the control group were $0.08,0.35,0.29$ and 0.37 for the four successive quartiles, with mean confidence ratings of 1.89 , $1.93,2.06$ and 2.11 respectively, and an overall mean of 2.00 . The mean confidence ratings to FAs for controls and VL did not differ statistically $(t<1.0)$. Thus relative to VL, control participants made many fewer FAs in this test, but expressed greater confidence in their errors. The main conclusion from the test however is that VL again showed a much greater FA rate than did age-matched control participants; it therefore seems that VL's tendency to make many false positives in recognition memory tests is not restricted to visually perceived items.

\subsection{Test 3. Face recognition memory}

In an attempt to gather further information about the types of material that evoked high false alarm rates in VL, we gave her a test of recognition memory for faces. In this task, we showed a series of faces, in close-up, presented on slides. VL and members of the control group sat in a comfortable chair and viewed the slides projected on an office wall. They were told that some faces would be repeated in a long series, and after viewing each face to say whether the face was new or a repeat. The slides were presented at a 5-s rate. The series consisted of 67 slides; 24 slides of faces were repeated exactly, 8 each at lags of 2, 10 and 20 intervening items, leaving 39 faces as possible false alarms. Mean hit rates for the control group were $0.80,0.64$ and 0.63 for lags 2,10 and 20 respectively, with an FA rate of 0.17 . VL's hit rates for lags 2,10 and 20 were $1.00,1.00$ and 0.88 respectively, with an FA rate of 0.49 . VL's performance level on hits was not different from the control hit rate (all $t$ values were 1.65 or less), but her FA rate was significantly greater than the controls $[t(11)=3.07, p<0.01]$. Her performance with faces was therefore similar to her performance with pictures and sounds-high hit rates, but also a very high false alarm rate.

\subsection{Test 4. Sentence recognition memory}

Following speculation that VL might show especially high FA rates to meaningful, rich mental representations, a sentence task was also designed to measure recognition of vivid, concrete sentences, as well as abstract sentences. The task consisted of 25 , rich easily imageable concrete sentences (e.g., 'The strict teacher suddenly banged the ruler on his table') and 25 abstract sentences, as devoid of imagery as possible (e.g., 'The content of the abstract sentence was almost entirely lacking'). The 50 sentences were presented visually in random order, in a list with 10 repeated concrete sentences and 10 repeated abstract sentences at lags ranging between 15 and 30 intervening sentences. Thus, in all, 70 sentences were presented $(50+20)$. Participants were asked to read each sentence, to rate each one 1-10 for how vivid an image it suggested, and then say whether it was new or a repeated sentence. Hit rates for concrete sentences were 0.92 for controls and 0.50 for $\operatorname{VL}[t(11)=4.04, p<0.001]$; for abstract sentences hit rates were 0.66 and 0.60 for controls and VL respectively $(t<1.0)$. The FA rates for concrete sentences were 0.02 and 0.12 for controls and VL respectively $[t(11)=4.80, p<0.001]$; for abstract sentences FA rates were 0.04 and 0.20 for controls and VL respectively $[t(11)=3.07, p<0.001]$. Thus, although VL showed lower hit rates and higher FA rates than control participants, her FA rates were numerically not as high as with pictorial materials. It had been speculated that VL might show especially high FA rates to meaningful, vividly rich representations. However, the results suggest that this was not the case, as FA rates were numerically higher for the less vivid, abstract sentences. This may reflect the novelty of the abstract sentences used, as the tone and meaning of such sentences might have been less familiar to VL. In summary, VL showed an extremely high FA rate to face stimuli, similar to the FA rates she showed to pictures and environmental sounds, but only moderate FA rates to sentences, similar to the FA rates she showed to visually presented words as described next.

\subsection{Test 5. Word recognition memory}

A continuous word recognition study was carried out with the primary objective of checking whether VL's high false alarm propensity would also extend to verbal materials. In addition, words were presented either visually (V) or auditorily (A) so that repeated words could be presented and tested either in the same modality (AA, VV) or across modalities (AV, VA). Lag was varied (5, 10,20 or 40 intervening items) to obtain information on forgetting rate. Finally, a number of low frequency distractors were included (e.g., gerund, tinder) to check whether VL tended to say 'yes' to everything, or whether she would discriminate between medium and low frequency distractors.

In outline, 169 words were presented either visually (computer screen) or auditorily (from a tape recorder). Presentation was selfpaced with a lower limit of $6 \mathrm{~s} /$ word; the experimenter pressed a key when the participant was ready to proceed. Words were mostly medium frequency concrete nouns, 5-8 letters. The target words (i.e., repeated words) were all medium frequency; there were three exemplars of each combination of the four modality pairs (AA, VV, AV, VA) with the four lags $(5,10,20,40)$, thus giving 48 exemplars and 96 items. In addition, 24 medium frequency distractors (non-repeated words) were presented auditorily and 24 visually; also 10 low frequency distractor words were presented auditorily and 10 visually; finally there were 5 primacy buffer items (thus $5+48+48+48+20=169$ words in total). All participants were asked to say whether each word had occurred before in the series.

The results (Table 2) showed that the modality of encoding and retrieval had no systematic effect; also that whereas controls showed a declining hit rate with increasing lag, VL's data did not. The table also shows that whereas VL's hit rates were lower than that of controls, her FA rate was higher, especially in the case of auditory distractor items: 0.42 compared to 0.18 for controls, although this difference was not reliable $[t(11)=1.54, p>0.10]$. Her tendency to make false alarms to words was thus numerically much smaller than for pictures or sounds. Strikingly, she made no false alarm errors to low frequency distractors, and this point is raised again in the Discussion. No clear patterns emerged from either the modality manipulation or the lag manipulation, perhaps because of the small number of exemplars (for VL especially). There is some suggestion that VL maintained a higher criterion (lower hit rate and FA rate) for visually presented test items of medium frequency: hit rates and FA rates were 0.66 and 0.16 , respectively, for visual test items, and 0.79 and 0.42 respectively for auditory test items. However, the main points from this experiment appear to be that, first, although VL shows a higher FA rate than controls to verbal items, the effect is much less than with pictures or sounds. Second, VL does not simply say 'yes' to everything-she gave no false alarms to low frequency words.

\section{General discussion}

We first summarize the salient features of VL's case. In terms of everyday observations, VL exhibited many instances of déjà vécu - 
erroneous recollections - in the course of her daily life. These instances would typically be triggered by a scene, a person or an incident that caught her attention; she commented on the events with mild surprise (e.g., "It's funny that the man is always standing on that same street corner when we pass by"), but with no attempt to correct herself rationally (e.g., "but I have never visited this town before, so I must be wrong"). Other than mild memory problems and some difficulties in performing routine tasks, VL seemed cognitively normal; her speech was fluent and coherent.

Neuropsychological testing showed that VL was suffering from mild to moderate dementia (MMSE $=18 / 30$ ) although her performance on the Mattis scale was in the normal range apart from the memory component. She was also judged to be 'low normal' on the WCST, but above average on phonemic fluency. Her performance was impaired on all memory tests, on Boston naming and semantic fluency, but was normal on tests of perception and speech. This pattern of strengths and weaknesses suggested damage to the MTL, and this was confirmed by the neuroimaging evidence. Her tendency to report erroneous recollections in daily life was echoed in the lab experiments, which showed that VL made many false alarms to new stimuli in recognition memory tasks. This very high false alarm rate was found with visual and auditory materials, with pictures, sounds, faces, words and sentences, although the false alarm rate was somewhat lower with verbal materials. We included some low frequency distractor words in the verbal tests, assuming that these unusual words would elicit a high number of false alarms; this did not happen, however (Table 2). We can thus say that VL's high false alarm rates did not reflect a simple tendency to say yes to all items, and speculate that her instances of déjà vécu to striking events in the environment were associated with unexpectedness rather than with rarity as such.

Finally, automated whole-brain volumetry provided evidence of frontal lobe damage in both left and right lateral regions, as well as damage to anterior and posterior temporal regions, predominantly on the right (Fig. 2). Further hand-traced volumetric assessment of VL's MTL structures gave evidence of extensive tissue loss in both right and left hippocampus (numerically more loss on the left), and also in both right and left parahippocampal cortex (again numerically more on the left, Fig. 3). VL's tissue losses were thus primarily in frontal and MTL regions, with damage in the latter being mainly in hippocampus and parahippocampal cortex. These areas of damage are broadly in line with VL's general cognitive profile - extensive memory and naming problems - although one might have expected more behavioral evidence of executive function deficits given her frontal damage. Speculatively, her excellent performance on the phonemic fluency test may be attributable to her well-preserved verbal functions (see her high vocabulary and speech scores in Table 1). Additionally, her WCST scores do show some impairment, in line with the observed frontal lobe damage.

How can we link evidence from the various experimental, neuropsychological and structural imaging tests to VL's bizarre errors of false recollection? Most accounts of déjà vu and déjà vécu take the position that the erroneous feelings reflect a disorder of perceptual processing. The normal processes of perception are combined with abnormal feelings of familiarity, probably associated with random firing of MTL structures, and this combination results in the experience of déjà vu (e.g., Bancaud et al., 1994; Spatt, 2002). In the case of déjà vécu, the feeling of intense familiarity is interpreted falsely as a genuine recollection, either because of a mismatch between MTL and frontal processing (O'Connor et al., 2010) or because of a further process of recollective confabulation, in which the cognitive system erroneously constructs a plausible scenario to resolve the difference between perceptual novelty and the feeling of familiarity (Moulin 2013;
Moulin et al., 2005). In these accounts there is little or no role for memory processes, yet a number of investigators, starting with Penfield and Perot (1963) have reported that their patients experienced "vivid visual perceptions of familiar scenes" (Barbeau et al., 2005) or "memories from earlier life" (Bancaud et al., 1994; Bartolomei et al., 2004; Hamani et al., 2008). These evoked memories were typically generic in character, and did not involve specific episodes, yet they nonetheless suggest some involvement of records of past experiences with current processing.

Barense and colleagues have recently shown how deficits in perceptual processing can result in an apparent memory failure (Barense et al., 2012; Newsome, Duarte, \& Barense, 2012). They compared the performance of amnesic patients and healthy controls on a visual pattern-matching task in which two visual objects were presented simultaneously; the participant's task was to decide whether the two stimuli were identical or mismatched. The results showed that when the stimuli used were complex and ambiguous, performance of the amnesic patients with lesions to the perirhinal cortex declined precipitously in the second half of the test condition. The researchers' explanation built on a representational-hierarchical account of visual processing in the ventral stream (Desimone \& Ungerleider, 1989; Bussey, Saksida, \& Murray, 2002), which suggests that visual information flows from posterior to anterior brain areas in increasingly complex conjunctions, culminating in representations of objects in the perirhinal cortex. Individuals with intact perirhinal processing can readily decide whether or not two objects are identical, but patients with perirhinal damage are forced to rely on lower levels of visual analysis, and will make errors in situations where there is substantial overlap in features between current items and previously processed items. As an example, if a current item is represented as $A B C$ at the object level, its constituent feature representations at a lower feature level might be $A, B$, and $C$. If a recently presented item was $A B D$, its constituent feature representations might be $A, B$, and $D$, thus overlapping at the featurelevel with the current item $A B C$. Assuming that some memory of prior feature analysis persists at lower levels of visual processing (e.g., memory for features A, B, and D), decisions about the identity of current items (e.g., object $A B C$ ) will get progressively more difficult for individuals with perirhinal damage-because the fragmented feature representations from previous analyses build up and interfere with processing subsequent items. This intact feature-level memory, combined with impaired object-level memory, can lead to false recognition of objects containing overlapping features (McTighe, Cowell, Winters, Bussey, \& Saksida, 2010; Romberg et al., 2012; Yeung, Ryan, Cowell, \& Barense, 2013).

Although VL may have had some damage to her perirhinal cortex, evidence from her structural scan suggests that structures at a higher level (hippocampus and parahippocampal cortex) were likely more affected (Fig. 3). Barense et al. (2012) point out that a similar argument to their perceptual interference hypothesis can apply to hippocampus and parahippocampal cortex, albeit at a higher level of analysis-such as spatial scenes, and the spatiotemporal contexts comprising episodes (see also Lee et al. (2005a, 2005b)). We propose therefore that individuals (such as VL) with substantial damage to the hippocampus and parahippocampal cortex will experience analogous difficulties to those with perirhinal damage, but in this case for spatial, contextual, and episodic information, due to the necessary reliance on lower levels of analysis and to the buildup of interference from prior processing. That is, in the absence of a fully functioning parahippocampal cortex and hippocampus, the elements that comprise scenes, contexts, and events are poorly bound, such that a novel episode may erroneously feel familiar, due to the fact that the elements comprising the event are indeed familiar. In other words, the daily events of everyday life (visiting the doctor, going shopping) 
contain many overlapping elements (e.g., being picked up by your daughter, driving in the car, passing the same bus stop). When these elements are not bound into unique event representations, a truly novel event may in fact feel familiar-because it is comprised of truly familiar elements.

There is growing agreement that one major function of the parahippocampal cortex is the representation of contextual information, and that the hippocampus is the locus of binding item and contextual information to form an encoded event (reviewed by Diana et al. (2007), Ranganath and Ritchey (2012)). These authors propose that, during encoding, information about visual objects is analyzed at various levels in the visual system and is finally represented in the perirhinal cortex. In a complementary fashion, information about context is represented in the parahippocampal cortex, and the two types of information are then bound together in the hippocampus to form an encoded event. In a later recognition test, perception of a face or other complex object is again represented in the perirhinal cortex, signaling familiarity for that face or object (Diana et al., 2007; Ranganath \& Ritchey, 2012). During recognition, the current context will also be analyzed and represented in the parahippocampal cortex, as will the conjunction of item and context information in the hippocampus. Dynamic interactions among these three regions (see Barbeau et al. (2005), Diana et al. (2007)) may also lead to retrieval of cortically-stored representations of similar past events, and (speculatively) be associated with the experience of recollection.

VL's major memory problems were first an extremely high false alarm rate on laboratory-based tasks with a variety of visual, auditory and verbal materials. Second, she experienced and demonstrated frequent instances of déjà vécu in everyday life, typically triggered by some event that caught her attention. As detailed previously, her major areas of atrophy included hippocampus, parahippocampal cortex and lateral frontal regions-all bilaterally. Thus, when some salient object, person or event caught VL's attention, the information would be processed up to the level of the perirhinal cortex and parahippocampal cortex. In the healthy brain, the conjunction of a new object and context would be bound together as a unique and cohesive representation of the novel event, but this binding was greatly impaired in VL's case. We suggest that in VL elements of these poorly-bound conjunctions of item and context would occasionally overlap with similar but not identical elements of previous events-thereby giving rise to erroneous "recollections" of the current event. That is, her erroneous recollections may have been driven by fragmented event representations, such that false memory for the novel current episode was triggered by accurate memory for its elements. In the words of O'Connor et al. (2010) the current encoding was occasionally experienced as retrieval. Normally this erroneous recollection would be edited out by retrieval monitoring processes in the right frontal lobe (Shallice et al., 1994; Rugg, Fletcher, Frith, Frackowiak, \& Dolan, 1996), but right frontal areas were damaged in $\mathrm{VL}$, so the retrieval check failed to operate, and she reported 'recollecting' the present event with no surprise or comment. Given that VL showed atypically high recognition false alarm rates to auditory as well as to visual items, it is necessary to conclude that her problems were not associated specifically with visual processing, but rather occurred at a higher level of analysis.

This account is similar to the model of déjà vécu proposed by O'Connor et al. (2010), and is compatible with the proposals of Barbeau et al. (2005) to describe the effects of stimulating the perirhinal region. Both groups also stress the importance of dynamic interactions among relevant brain regions in the MTL and visual cortex when these interactions are synchronized in the theta range of brain electrical activity. Information on this latter point is unfortunately not available in the case of $\mathrm{VL}$, but we have no reason to discount its crucial importance.
Our present proposals rely more on the idea that, owing to the extensive damage to the hippocampus and parahippocampal cortex, VL's experience of events was often based on fragmented event representations. Thus, in parallel with the findings of Barense et al. (2012) and Yeung et al. (2013), VL's pathologically high false alarm rates in recognition memory experiments may have stemmed from the overlap of features from the present stimulus array with features from previous items, thereby giving rise to feelings of "pastness." In her everyday life, the same faulty mechanism arguably gave rise to erroneous conjunctions of present and past representations, resulting in false "recollections" of novel present events. A critical feature of déjà vu experiences is the intense feeling of familiarity combined with the rational knowledge that the situation should not feel familiar. In the case of VL, however, the vivid sense of familiarity generated by faulty processing of damaged MTL structures was not 'corrected' by later stages of processing, owing to damage to her right frontal lobe. In our view, it is this combination of MTL and frontal damage that results in the disorder of false recollection.

The case history observations of VL's erroneous recollections are strikingly similar to those reported from other patients by Moulin (2013) and others. Given this similarity, are there reasons to favor our suggestion that the incidents reflect feelings of familiarity emerging from fragmented event representations that are not appropriately edited by retrieval monitoring processes over other accounts in terms of faulty outputs from the hippocampus (O'Connor et al., 2010) or as recollective confabulations (Moulin, 2013)? We argue that the very high false alarm rates observed in VL's picture recognition behavior are similar to the perceptual interference effects shown by amnesic patients (Barense et al., 2012) and by patients with MCI (Newsome et al., 2012). The further observation that VL made progressively more false alarms (with increasing confidence) in both picture and sound recognition (Tests 1 and 2) as the testing session continued is also in line with the results of Barense et al. (2012), who found a similarly increasing pattern of perceptual errors as the test series continued in amnesic patients with MTL damage. This quartile analysis was not possible for the control group in the picture recognition test (Test 1 ) as they made very few FA errors. In the sound recognition test (Test 2), controls' FA rate increased from 0.08 to 0.37 (a difference of 0.29 ), compared to VL's increase from 0.32 to 0.71 (a difference of 0.39 ). In that same test, the control group's confidence ratings increased from 1.89 to 2.11 from first to fourth quartile (a difference of 0.22 ) compared to VL's corresponding increase from 1.33 to 1.90 (a difference of 0.57). Thus FA rates and confidence ratings increased across quartiles in both controls and in VL, although VL's increases were somewhat greater in magnitude.

We were also able to compare VL's mean confidence ratings to hits and FAs in the picture and sound recognition tests to those of the control group. Interestingly, all four comparisons showed that VL's mean confidence levels were lower than the corresponding control levels; 2.61 vs. 2.98 for picture hits, 1.81 vs. 1.93 for picture FAs, 2.00 vs. 2.55 for sound hits, and 1.65 vs. 2.00 for sound FAs, although only the comparison for picture hits was statistically significant. Confidence levels for hits were understandably higher than confidence levels for FAs, but given VL's apparent confidence in her real-life erroneous recollections, it is surprising that her confidence levels were relatively low for FAs in these laboratory tests. This result may be speculatively attributed to the fact that she knew her memory was being tested, and also knew that her memory was poor, so was somewhat tentative in her judgments. In real-world situations she had perhaps no reason to doubt the validity of her perceived recollections.

The picture recognition test also included the question "have you ever seen this picture before?" to check whether participants 
had seen specific pictures previously in magazines. VL claimed to have seen $20 \%$ of the pictures before, as opposed to $3 \%$ of the pictures by a group of comparable older adults-again suggesting that current processing was erroneously conjoined to similar records of previously viewed pictures. The finding that VL also made many false alarms in an auditory recognition test makes it clear that her problems were not associated specifically with visual processing but appeared to occur at a higher level of analysis. It is of course possible that her episodes of déjà vécu in real life reflected a different processing error from the problems associated with her high false alarm rates, but it is more parsimonious to assume one common explanation for her various instances of erroneous recollection.

One surprising aspect of the present results is the finding that VL's FA rate was much larger for picture recognition (0.79) than it was for verbal materials (average of 0.16 for sentences and average of 0.29 for medium frequency words). This pattern contrasts strongly with the results of the control group who had FA rates of 0.01 for pictures, 0.03 for sentences and 0.16 for medium frequency words. The finding of much better recognition discrimination for pictures than for words in the control group (Table 2) is in line with the picture superiority effect-pictures are typically better remembered than words, arguably because of the greater distinctiveness of pictorial relative to verbal features (e.g., Nelson (1979)). Why was this typical effect reversed so dramatically in VL's case? One relevant study was reported by Wong, Cramer, and Gallo (2012). These investigators had participants study both words (presented in either red or blue font) or as pictured objects (presented either in full color or as line drawings); at test, participants were shown pairs of black words and had to choose either the word originally presented in blue font (font test) or the word originally studied as a colored picture (picture test). They also rated confidence in their choice from 50 (chance) to 100 (certain). Combining their results with those from previous studies the authors concluded that "errors are overall less likely for pictures than font color.... but when errors do occur, older adults are more likely than younger adults to make high confidence errors for pictures relative to font color, potentially because pictures have more features to erroneously recombine into a false recollection" (Wong et al., 2012, p. 1064). The suggestion that VL may also have recombined pictorial features to yield false recollections is an attractive one, although her false positive errors in the picture test were not given with unusually high confidence; her mean confidence rating was 1.81 compared to 1.93 for the control group. Thus VL's pattern of errors does not appear to correspond to the pattern shown by older adults reported by Wong et al. (2012). Similarly, on our test of sound recognition, VL's mean confidence rating was 1.58 compared to 2.00 for the control group, suggesting that although VL made many more false alarm errors, they were not made with greater confidence relative to the control group.

Other possible accounts of VL's comparatively low FA rates for verbal materials (Table 2) follow from the fact that her verbal abilities were generally well preserved-at least in terms of everyday speech and relatively 'well-supported' verbal tasks such as the NART and WAIS Vocabulary. As a speculation, in her case words may have been encoded in a relatively distinctive manner compared with pictures, which were abnormally encoded at the feature-level, rather than the object-level (McTighe et al., 2010; Romberg et al., 2012; Yeung et al., 2013), resulting in later confusions and FAs.

How does VL's pattern of memory impairments, especially her extremely high false alarm rates, compare with other cases in the literature? With regard to other cases of Alzheimer's disease (AD) Budson, Daffner, Desikan, and Schacter, 2000; Budson, Sullivan, Mayer, Daffner, Black, \& Schacter, 2002) reported that AD patients showed high false alarm rates to unrelated words in the DRM paradigm (Roediger \& McDermott, 1995) although the patients did not make more false alarms than control participants to related lures (e.g., to "smoke" after studying a list containing such words as cigarette, puff, cigar and ashes). In Budson et al. (2000), AD patients' FA rate to unrelated lures was 0.28 compared to 0.04 in older adults; in Budson et al. (2002)) the FA rates were 0.42 and 0.05 for AD patients and older adults respectively. These numbers are certainly comparable to the FA rates shown by VL for word recognition (Table 2, Test 5), although it should be recalled that VL's FA rate for other materials was substantially higher. We can also report that during informal pilot testing of the present picture recognition list we tested two early $\mathrm{AD}$ patients aged 60 and $70 \mathrm{yr}$ respectively. Their average hit rate was 0.76 (compared to 1.00 for VL) and their average FA rate was 0.03 (compared to 0.79 for VL). Additionally, there appears to be little or no evidence for elevated rates of déjà vécu in $A D$ patients, leading to the tentative conclusion that VL's problems are not directly associated with her diagnosis of $A D$.

Patients with lesions of the frontal cortex form another group showing elevated rates of false recognition. For example, Budson et al. (2002) reported an FA rate of 0.17 for unrelated lures on the first test trial of the DRM paradigm in a group of 13 patients with lesions to the frontal cortex. This figure contrasted with an FA rate of 0.05 in a matched control group. Other single-case studies of patients with frontal lesions include an account of patient BG reported by Schacter, Curran, Galluccio, Milberg, and Bates (1996). This patient had an infarction of the right frontal lobe and, like VL, showed pathological high FA rates in recognition memory for visual words, auditory words, pseudo words, pictures and environmental sounds. In a striking demonstration, the investigators found that the patient's high FA rate was eliminated when presented with categorized lists of words to study and then tested with lures from non-presented categories. His problems were attributed to an over-reliance on memory for general characteristics of encoded stimuli together with an impairment in memory for the specific features that allow unimpaired individuals to reject lures. Unfortunately we did not carry out the non-categorized item test on VL, although the pictures used in Test 1 were all rather distinctive, as demonstrated by the FA rate of 0.01 shown by the control group of older adults.

Another aspect of BG's false alarms was that he largely rated items as 'recollected' (R) as opposed to merely 'Known' (K). The combination of a high FA rate with high confidence was also found in case JB reported by Parkin, Bindschaedler, Harsent, and Metzler (1996). This patient had a left frontal lesion and showed a very high FA rate for words with most errors rated as "sure" he had studied them before, although in a second test he marked all FAs as known (K) as opposed to recollected (R). Finally, Delbecq-Derouesné, Beauvois, and Shallice (1990) reported results of recognition tests in a patient (RW) with bilateral areas of frontal hypodensity. This patient also showed abnormally high FA rates, and in a test of face recognition rated $75 \%$ of his erroneous responses as "certain" that he was correct. The pattern of false alarm errors shown by VL is somewhat similar to those shown by frontal patients, but with the exception that her responses were made with confidence ratings lower than controls. This is in contrast to her instances of false recollection made in real-life settings where she showed every indication of being quite sure of her observations. When discussing patient RW, Delbecq-Derouesné et al. (1990) made the interesting suggestion that his behavior showed "an inability to distinguish the experience of remembering from another kind of misleading subjective experience triggered by the distractors" (p. 1069). In other words, perception and remembering were occasionally confused by the patient, an observation similar to the suggestion by O'Connor et al. (2010) that encoding is occasionally interpreted as retrieval, and in line with the similarity between perceiving and remembering processes emphasized by Barense et al. (2012) and Craik (2002) 
among others. This type of confusion appears to fit the pattern shown by VL in everyday life, as well as in the lab results. A cautious conclusion could be that her erroneous experiences were attributable to the combination of medial-temporal as well as frontal degeneration.

In summary, the facts of VL's case are that she exhibited memory problems and frequent instances of déjà vécu experiences in her day-to-day life. On further investigation she showed extremely high false alarm rates on a variety of recognition memory tests-auditory and verbal as well as visual. Neuropsychological examination provided strong evidence of damage to MTL regions, and this damage - especially to the hippocampus and parahippocampal cortex - was confirmed by volumetric analyses of structural MRI data. These latter analyses also showed damage to lateral frontal areas. From these facts we have proposed a speculative account of VL's experiences of déjà vécu, but an account that is in line with previous reports of erroneous recollections.

\section{Acknowledgements}

This article was prepared with the help of grants to FIMC and MDB from the Natural Sciences and Engineering Research Council of Canada. We are grateful to Drs. Morris Freedman, Stefan Köhler and Kelly Murphy for very useful information and advice, and to Karen Lau and Alicia McNeely for help with preparation of the manuscript.

\section{References}

Aggleton, J. P. \& Brown, M. W. (1999). Episodic memory, amnesia and the hippocampal-anterior thalamic axis. Behavioral and Brain Sciences, 22(3), 425-444.

Bancaud, J., Brunet-Bourgin, F., Chauvel, P., \& Halgren, E. (1994). Anatomical origin of déjà-vu and vivid memories in human temporal-lobe epilepsy. Brain, 117 71-90.

Barbeau, E., Wendling, F., Regis, J., Duncan, R., Poncet, M., Chauvel, P., et al. (2005) Recollection of vivid memories after perirhinal region stimulations: Synchronization in the theta range of spatially distributed brain areas. Neuropsychologia, 43, 1329-1337.

Barense, M. D., Groen, I. I. A., Lee, A. C. H., Yeung, L. -K., Brady, S. M., Gregori, M., et al. (2012). Intact memory for irrelevant information impairs perception in amnesia. Neuron, 75, 157-167.

Bartolomei, F., Barbeau, E., Gavaret, M., Guye, M., McGonigal, A., Regis, J., et al. (2004). Cortical stimulation study of the role of rhinal cortex in déjà vu and reminiscence of memories. Neurology, 63, 858-864.

Bowles, B., Crupi, C., Mirsattari, S. M., Pigott, S. E., Parrent, A. G., Pruessner, J. C., et al (2007). Impaired familiarity with preserved recollection after temporal-lobe resection that spares the hippocampus. Proceedings of the National Academy of Sciences of the United States of America, 104, 16382-16387.

Bowles, B., Crupi, C., Pigott, S., Parrent, A., Wiebe, S., Janzen, J., et al. (2010). Double dissociation of selective recollection and familiarity impairments following two different surgical treatments for temporal-lobe epilepsy. Neuropsychologia, 48 , 2640-2647.

Brown, A. S. (2003). A review of the déjà vu experience. Psychological Bulletin, 129, 394-413.

Brown, A. S. (2004). The déjà vu illusion. Current Directions in Psychological Science, 13(6), 256-259.

Budson, A. E., Daffner, K. R., Desikan, R., \& Schacter, D. L. (2000). When false recognition is unopposed by true recognition: Gist-based memory distortion in Alzheimer's disease. Neuropsychology, 14, 277-287.

Budson, A. E., Sullivan, A. L., Mayer, E., Daffner, K. R., Black, P. M., \& Schacter, D. L (2002). Brain, 125, 2750-2765.

Bussey, T. J., Saksida, L. M., \& Murray, E. A. (2002). Perirhinal cortex resolves feature ambiguity in complex visual discriminations. European Journal of Neuroscience $15,365-374$.

Callen, D. J. A., Black, S. E., Gao, F., Caldwell, C. B., \& Szalai, J. P. (2001). Beyond the hippocampus-MRI volumetry confirms widespread limbic atrophy in AD. Neurology, 57(9), 1669-1674.

Craik, F. I. M. (2002). Levels of processing: Past, present .... and future? Memory, 10 , 305-318.

Crawford, J. R., Garthwaite, P. H., \& Howell, D. C. (2009). On comparing a single case with a control sample: An alternative perspective. Neuropsychologia, 47 . 2690-2695.
Dade, L. A., Gao, F. Q., Kovacevic, N., Roy, P., Rockel, C., O’Toole, C. M., et al. (2004). Semiautomatic brain region extraction: A method of parcellating brain regions from structural magnetic resonance images. NeuroImage, 22(4), 1492-1502.

Delbecq-Derouesné, J., Beauvois, M. F., \& Shallice, T. (1990). Preserved recall versus impaired recognition. Brain, 113, 1045-1074.

Desimone, R., \& Ungerleider, L. G. (1989). Neural mechanisms of visual processing in monkeys. In: F. Boller, \& J. Grafman (Eds.), Handbook of neuropsychology (pp, 267-299). New York: Elsevier Science.

Diana, R. A., Yonelinas, A. P., \& Ranganath, C. (2007). Imaging recollection and familiarity in the medial temporal lobe: A three-component model. Trends in Cognitive Sciences, 11, 379-386.

Dywan, J., \& Jacoby, L. L. (1990). Effects of aging on source monitoring: Differences in susceptibility to false fame. Psychology and Aging, 5, 379-387.

Gardiner, J. M., \& Richardson-Klavehn, A. (2000). Remembering and knowing. In: E. Tulving, \& F. I. M. Craik (Eds.), The Oxford handbook of memory (pp. 229-244). New York: Oxford University Press.

Glisky, E. L., Polster, M. R., \& Routhieaux, B. C. (1995). Double dissociation between item and source memory. Neuropsychology, 9(2), 229-235.

Guedj, E., Aubert, S., McConigal, A., Mundler, O., \& Bartolomei, F. (2010). Déjà vu in temporal lobe epilepsy: Metabolic pattern of cortical involvement in patients with normal brain MRI. Neuropsychologia, 48, 2174-2181, http://dx.doi.org/ 10.1016/j.neuropsychologia.2010.04.009.

Hamani, C., McAndrews, M. P., Cohn, M., Oh, M., Zumsteg, D., Shapiro, C. M., et al. (2008). Annals of Neurology, 63(1), 119-123.

Jacoby, L. L. (1991). A process dissociation framework-Separating automatic from intentional uses of memory. Journal of Memory and Language, 30(5), 513-541.

Jacoby, L. L., \& Whitehouse, K. (1989). An illusion of memory-False recognition influenced by unconscious perception. Journal of Experimental Psychology: General, 118(2), 126-135.

Kovacevic, N., Lobaugh, N. J., Bronskill, M. J., Levine, B., Feinstein, A., \& Black, S. E. (2002). A robust method for extraction and automatic segmentation of brain images. Neurolmage, 17(3), 1087-1100.

Lee, A. C., Buckley, M. J., Pegman, S. J., Spiers, H., Scahill, Gaffan D., \& Graham, K. S. (2005a). Specialization in the medial temporal lobe for processing of objects and scenes. Hippocampus, 15, 782-797.

Lee, A. C., Bussey, T. J., Murray, E. A., Saksida, L. M., Epstein, R. A., Kapur, N., et al. (2005b). Perceptual deficits in amnesia: Challenging the medial temporal lobe 'mnemonic' view. Neuropsychologia, 43, 1-11.

Levy-Cooperman, N., Ramirez, J., Lobaugh, N. J., \& Black, S. E. (2008). Misclassified tissue volumes in Alzheimer disease patients with white matter hyperintensities-Importance of lesion segmentation procedures for volumetric analysis. Stroke, 39, 1134-1141.

Mandler, G. (1980). Recognizing-The judgement of previous occurrence. Psychological Review, 87, 252-271

Martin, C. B., Mirsattari, S. M., Pruessner, J. C., Pietrantonio, S., Burneo, J. G., Hayman-Abello, B., et al. (2012). Déjà vu in unilateral temporal-lobe epilepsy is associated with selective familiarity impairments on experimental tasks of recognition memory. Neuropsychologia, 50, 2981-2991, http://dx.doi.org/ 10.1016/j.neuropsychologia.2012.07.030.

McTighe, S. M., Cowell, R. A., Winters, B. D., Bussey, T. J., \& Saksida, L. M. (2010). Paradoxical false memory for objects after brain damage. Science, 330(6009), $1408-1410$.

Moulin, C. J. A. (2013). Disordered recognition memory: Recollective confabulation. Cortex, 49, 1541-1552.

Moulin, C. J. A., Conway, M. A., Thompson, R. G., James, N., \& Jones, R. W. (2005). Disordered memory awareness: Recollective confabulation in two cases of persistent déjà vécu. Neuropsychologia, 43(9), 1362-1378.

Nelson, D. L. (1979). Remembering pictures and words: Appearance, significance, and name. In: L. S. Cermak, \& F. I. M. Craik (Eds.), Levels of processing in human memory (pp. 45-76). Hillsdale, NJ: Lawrence Erlbaum Associates.

Newsome, R. N., Duarte, A., \& Barense, M. D. (2012). Reducing perceptual interference improves visual discrimination in mild cognitive impairment: Implications for a model of perirhinal cortex function. Hipppocampus, 22, 1990-1999.

O'Connor, A. R., Lever, C., \& Moulin, C. J. A. (2010). Novel insights into false recollection: A model of déjà vécu. Cognitive Neuropsychiatry, 15, 118-144.

Parkin, A. J., Bindschaedler, C., Harsent, L., \& Metzler, C. (1996). Pathological false alarm rates following damage to the left frontal cortex. Brain $\mathcal{E}$ ' Cognition, 32, $14-27$

Penfield, W., \& Perot, P. (1963). The Brain's record of auditory and visual experience -A final summary and discussion. Brain, 86, 595-696.

Ramirez, J., Gibson, E., Quddus, A., Lobaugh, N. J., Feinstein, A., Levine, B., et al. (2011). Lesion explorer: A comprehensive segmentation and parcellation package to obtain regional volumetics for subcortical hyperintensities and intracranial tissue. Neurolmage, 54, 963-973.

Ramirez, J., Scott, C. J. M., \& Black, S. E. (2013). A short-term scan-rescan reliability test measuring brain tissue and subcortical hyperintensity volumetrics obtained using the lesion explorer structural MRI processing pipeline. Brain Topography, 26, 35-38.

Ranganath, C., \& Ritchey, M. (2012). Two cortical systems for memory-guided behaviour. Nature Reviews Neuroscience, 13, 713-726.

Roediger, H. L., \& McDermott, K. B. (1995). Creating false memories: Remembering words not presented in lists. Journal of Experimental Psychology: Learning, Memory, and Cognition, 21, 803-814. 
Romberg, C., McTighe, S. M., Heath, C. J., Whitcomb, D., Cho, K., Bussey, T. J., et al. (2012). False recognition in a mouse model of Alzheimer's disease: Rescue with sensory restriction and memantine. Brain, 135, 2103-2114.

Rugg, M. D., Fletcher, P. C., Frith, C. D., Frackowiak, R. S. J., \& Dolan, R. J. (1996). Differential activation of the prefrontal cortex in successful and unsuccessful memory retrieval. Brain, 119, 2073-2083.

Rugg, M. D., \& Yonelinas, A. P. (2003). Human recognition memory: A cognitive neuroscience perspective. Trends in Cognitive Sciences, 7, 313-319.

Schacter, D. L., Curran, T., Galluccio, L., Milberg, W. P., \& Bates, J. F. (1996). False recognition and the right frontal lobe: A case study. Neuropsychologia, 34 793-808.

Shallice, T., Fletcher, P., Frith, C. D., Grasby, P., Frackowiak, R. S. J., \& Dolan, R. J. (1994). Brain regions associated with acquisition and retrieval of verbal episodic memory. Nature, 368, 633-635.
Spatt, J. (2002). Déja vu: Possible parahippocampal mechanisms. Journal of Neuropsychiatry and Clinical Neurosciences, 14(1), 6-10.

Tulving, E. (1973). Episodic and semantic memory. In: E. Tulving, \& W. Donaldson (Eds.), Organization of memory (pp. 381-403). New York: Academic Press.

Tulving, E. (1985). How many memory-systems are there? American Psychologist, $40,385-398$.

Wong, J. T., Cramer, S. J., \& Gallo, D. A. (2012). Age-related reduction of the confidence-accuracy relationship in episodic memory: Effects of recollection quality and retrieval monitoring. Psychology and Aging, 27, 1053-1065.

Yeung, L. K., Ryan, J. D., Cowell, R. A., \& Barense, M. D. (2013). Recognition memory impairments caused by false recognition of novel objects. Journal of Experimental Psychology: General

Yonelinas, A. P. (2002). The nature of recollection and familiarity: A review of 30 years of research. Journal of Memory and Language, 46(3), 441-517. 